\title{
FAST NEUTRON DETECTORS BASED ON MICROMEGAS TECHNOLOGY
}

\section{S. Andriamonje *}

CEA-Saclay, DSM/DAPNIA

F- 91191 Gif-sur-Yvette, France

E-mail: sandriamonjeecea.fr

\section{Giomataris and J. Pancin}

CEA-Saclay, DSM/DAPNIA

F- 91191 Gif-sur-Yvette, France

E-mail: Ioanis.Giomatarisecern.ch and jpancin@dapnia.cea.fr

\begin{abstract}
After a short description of the Micromegas principle, a new concept of neutron detectors based on this technique is presented. The report is illustrated by an overall picture of the possible use of these detectors in different domain such as: nuclear physics, inertial fusion and industrial application. A particular description will be devoted to the compact detector named "PiccoloMicromegas". This detector, able to measure neutron flux in a broad range of energy of neutron (from thermal to several $\mathrm{MeV}$ ), is developed for the measurements of neutrons flux in-core of the future generations of the nuclear reactors (fast and possibly Accelerator Driven System $(\mathrm{ADS}))$.
\end{abstract}

International Workshop on Fast Neutron Detectors

University of Cape Town, South Africa

April 3 - 6, 2006

\footnotetext{
${ }^{*}$ Speaker
} 


\section{Introduction}

Micromegas is a gaseous detector that has been developed initially for tracking in high rate high-energy experiments [1]. At present, Micromegas detector is used in many experiments $[2,3]$ and due to its high performances is being employed for searching rare events such as the Solar axion CAST [4].

The detection principle is simple: the gas volume is separated in two regions by a thin micromesh, the first one where the conversion and drift of the ionization electrons, occur and the second one, 50-160 micron thick, where the amplification takes place. Ionization electrons are created by the energy deposition of an incident charged particle in the conversion gap. In the amplification region, a high field ( 40 to $70 \mathrm{kV} / \mathrm{cm}$ ) is created by applying a voltage of a few hundred volts between the micromesh and the anode plane, which collects the charge produced by the avalanche process. The anode can be segmented into strips or pads. The positive ions are drifting in the opposite direction and are collected on the micromesh.

One of the main advantages of Micromegas is its robustness and its high resistance to radiations. These qualities have been exploited to develop a new neutron detector and in particular for the use in nuclear reactor environment: operation with very high neutron flux, high gamma ray background and possibly at high temperature.

After a description of the principle of the Micromegas for neutron detection, we give some examples of his application for neutron beam characterisation, for neutron tomography and for diagnostics of inertial fusion program.

A special attention will be given in the description of the new detector dedicated for the measurement of neutron flux in-core of nuclear reactor. In order to be placed inside an empty rod of a reactor the detector needs to be compact, sealed and very small $(3.5 \mathrm{~cm} \times 3.5 \mathrm{~cm} \times 3.5$ $\mathrm{cm}$ ), contrary to the usual Micromegas detector used in particle physics experiments hence the origin of the "Piccolo-Micromegas" name.

The advantage of this detector over conventional neutron flux detectors and the results obtained with the first prototype with $14 \mathrm{MeV}$ neutron source and inside a TRIGA reactor are presented.

\section{Description of Micromegas for neutron detection}

The Micromegas technology has been extended to develop a new neutron detector $[5,6]$. As mentioned before the principle is based on the detection of the electron created by ionization of the filled gas by charged particles. In order to operate the Micromegas detector as a neutron detector, appropriate neutron/charged particle converter must be employed which can be either the detector's filling gas or target with appropriate deposit on its entrance window.

Inter-dependent parameters such as the high neutron reaction cross section, the low charged particles energy loss inside the converter, their subsequent energy-angular distribution and the range inside the filling gas has been considered and optimised. 
An appropriate neutron/charged particle solid converter has been chosen $\left({ }^{6} \operatorname{Li}(\mathrm{n}, \alpha)\right.$ and ${ }^{10} \mathrm{~B}(\mathrm{n}, \alpha)$ ) for neutron energy up to $1 \mathrm{MeV}$. The main advantage of the solid converter concept is a great simplicity of the structure and especially the excellent spatial $(<50 \mu \mathrm{m})$ and time resolution $(\sim \mathrm{ns})$ that is obtained by using small drift gaps. Such performance is not easy to obtain using gaseous converters, for instance ${ }^{3} \mathrm{He}$. The draw back is the lower efficiency of the thin solid converter for thermal neutrons, limited by the neutron $(n, \alpha)$ cross section and the thickness of the ${ }^{6} \mathrm{Li}$ layer, which must be shorter than the alpha particle range. However, Monte Carlo calculations show that an optimal thickness of $120 \mu \mathrm{m}\left({ }^{6} \mathrm{Li}\right.$ target $)$ is required in order to obtain an efficiency of about $25 \%$ for thermal neutrons at room temperature.

\subsection{Micromegas detector for CERN n_TOF beam profiler}

One of the first application of Micromegas for neutron detection is the determination of the characteristics of the $\mathrm{n}_{-}$TOF neutron beam at CERN [7]. Since the neutron energy range of the n-TOF facility extends from $1 \mathrm{eV}$ to $250 \mathrm{MeV}$, there is not a unique choice of an efficient converter.

The combination of $(n, \alpha)$ reaction and elastic scattering on the filling gas of the detector has been used for the determination of the neutron beam profile of the $n_{-}$TOF facility at CERN. The main characteristics of the $n \_$TOF detector and results can be fund in reference [7].

In summary the principle is shown in Figure 1a), the neutron/charged particle converter employed are: $\left({ }^{6} \mathrm{Li}(\mathrm{n}, \alpha)\right.$ for neutron energy up to $1 \mathrm{MeV}$ and $\mathrm{H}\left(\mathrm{n}, \mathrm{n}^{\prime}\right) \mathrm{H}$ and ${ }^{4} \mathrm{He}\left(\mathrm{n}, \mathrm{n}^{\prime}\right)^{4} \mathrm{He}$ for high energy neutron) which are the detector's filling gas. The anode strips (50 in total) have a pitch of $1.5 \mathrm{~mm}$ and are separated by $100 \mu \mathrm{m}$ gaps and connected on a fast current preamplifier with a rise time of $1 \mathrm{~ns}$ associated with an innovative Data Acquisition System based on $1 \mathrm{GHz}$ flash ADC with a rate of $180 \mathrm{MHz}$.

a)

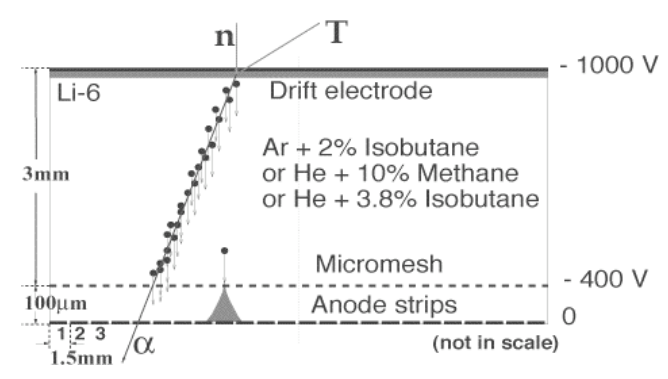

b)

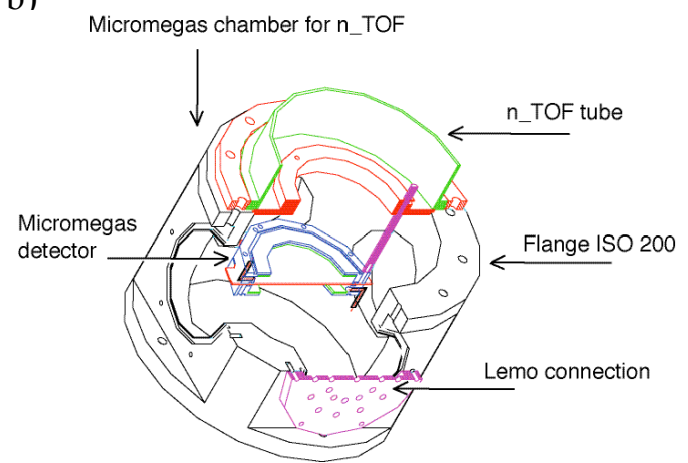

Figure 1: a) The principle of Micromegas for neutron detector used in $n+T O F$ experiment [7] b) Schematic view of the n_TOF Micromegas chamber

In order to achieve a very good profile of the n_TOF beam, the Micromegas detector has been placed in the vacuum chamber (Figure $1 \mathrm{~b})$ under the standard pressure of the $\mathrm{n}_{-}$TOF tube $\left(10^{-3}\right.$ bar). The main characteristics of this detector is the choice of material having a minimum 
influence on the high energy neutron. The example described here can be extended for other applications such as the diagnostic of the neutron beam for boron therapy [8] or hadron therapy [9].

\subsection{Micromegas detector for Inertial Confinement Fusion (ICF) experiments}

The development of Micromegas has extended recently by M. Houry et al [10] for a new neutron diagnostic to achieve high energy neutron spectroscopy in large gamma background.

This is the situation in the inertial confinement fusion (ICF). The concept of the detector is based on the association of a Micromegas detector with a solid neutron/charged particle converter. The DEMIN (DEtector MIcromegas for Neutron) detector is made of a neutron/charged particle conversion foil set in front of a Micromegas detector. The interaction of fast neutron in the neutron/charged particle converter produces charged particle which may be detected by the Micromegas detector. The principle of the concept is similar to the $\mathrm{n}_{-} \mathrm{TOF}$ Micromegas detector based on the detection of the recoil charged particle but the origin is not the same. For $\mathrm{n}_{-}$TOF it is from the filling gas. In DEMIN case, the neutrons also interact in the gas, but considering the difference of atomic density between the neutron/charged particle converter and the gas this second process is negligible. The schematic view of the DEMIN concept from reference [10] is reported in Figure 2. It shows in the figure the different thickness of the constituent of the detector: $2 \mathrm{~mm}$ for the polypropylene $\left(\mathrm{CH}_{2}\right)$ neutron/charged particle converter, $0.6 \mathrm{~mm}$ for the gaseous detector $(0.5 \mathrm{~mm}$ thick for the upper part and $0.1 \mathrm{~mm}$ for the bottom part).

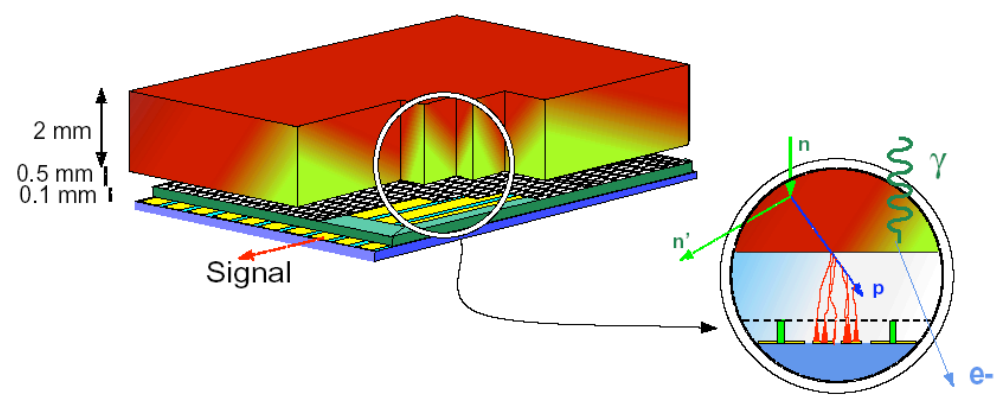

Figure 2: Schematic view of the DEMIN diagnostic. Ionization electrons are produced along the path of scattered protons and drift between the conversion foil and the epoxy substrate. On the other hand, electrons produced by $\gamma$-rays go through the gaps with a low ionization probability [10].

The thickness of the gas has been chosen thin enough to limit ionization by recoil electrons. Detailed description of the DEMIN detector and the experiments can be found in reference [10]. In summary, studies have been performed with a neutron generator and gamma-ray sources and it has been shown that $14 \mathrm{MeV}$ neutrons can be detected with improved efficiency and relative gamma insensitivity. This low gamma sensitivity makes this concept appealing for inertial fusion experiments. 


\subsection{Other Micromegas development for fast neutron detection}

The $\mathrm{n}_{-}$TOF Micromegas detector has been modified for the detection of fast neutrons from induced photofission [11]. A thin foil $12 \mu \mathrm{m}$ of polypropylene and the filling gas are used for neutron/charged particle converter. The development is for the characterisation of the radioactive parcel of nuclear waste. The method consists in an irradiation of the radioactive parcel by photons in order to create photofission reactions of the actinides present within the parcel. The detection of the fast neutron associated with fission could permit the determination of the quantity of the actinides contained in the waste panel. In this project the main quality of the needed detector is its very small sensitivity of high flux of gamma background. The experiment performed at Ghent University facility shows that Micromegas detector meets this requirement.

The Oak Ridge group [12] has also developed a new neutron detector based on Micromegas technology. The principle of the detector is quite similar to the one used for the $\mathrm{n} \_$TOF experiment $\left({ }^{6} \mathrm{Li}\right.$ and ${ }^{10} \mathrm{~B}$ are used for neutron/charged particle converter) but the technical fabrication is different for example the use of a quartz substracte using microfabrication techniques. The interesting work is the development of sealed 2D Micromegas detector which is very useful not only for the characterisation of the future neutron spallation source but also for other applications.

\section{Piccolo-Micromegas for neutron flux measurements in-core of nuclear reactor}

\subsection{Motivation}

Fast nuclear reactors and Accelerator Driven Systems (ADS) are considered as an alternative for energy production and transmutation of nuclear wastes. One important step needed for approval of a demonstrator is the experimental validation of simulations. Of particular interest is the determination of the neutron spectrum (i.e neutron flux as a function of the neutron energy) for different configurations of the subcritical device. As well known, the neutron flux in ADS consists of neutrons produced via spallation reactions in the target and fissions from the multiplying blanket.

Unfortunately the neutron spectra cannot be measured using only one type of detector. To cover the complete energy range of the produced neutrons, a new neutron detector concept based on Micromegas technology has been developed.

For illustration the possible subcritical configuration based in TRIGA-ADS project which consists of coupling a $1 \mathrm{MW}$ TRIGA reactor with a $140 \mathrm{MeV}$ proton accelerator has been chosen [13]. The core has a cylindrical shape, the fuel rods being arranged in seven concentric rings labeled A to $\mathrm{G}$. The central rod and the rods named ring B are reserved for the spallation target and its cooling system. The measurement can be only achieved starting from ring $\mathrm{C}$. The 
distribution of neutrons in three-dimensional space as a function of energy and time is simulated using innovative simulation codes (FLUKA and EA-MC [14,15] at CERN, and MCNP-4C [16] at ENEA/Casaccia).

An example of the neutron energy spectrum for different regions of the core for $\mathrm{k}_{\mathrm{src}}=0.97$ is given in Figure $4 \mathrm{~b}$. The measurement of neutron flux spectra is a necessary step in order to characterize the neutronics of the system.

a)

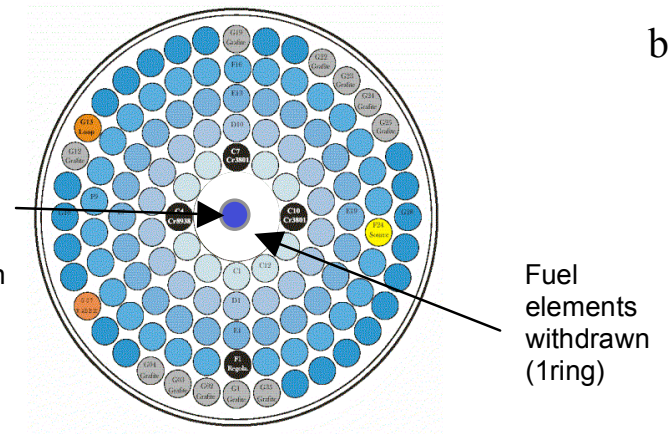

b)

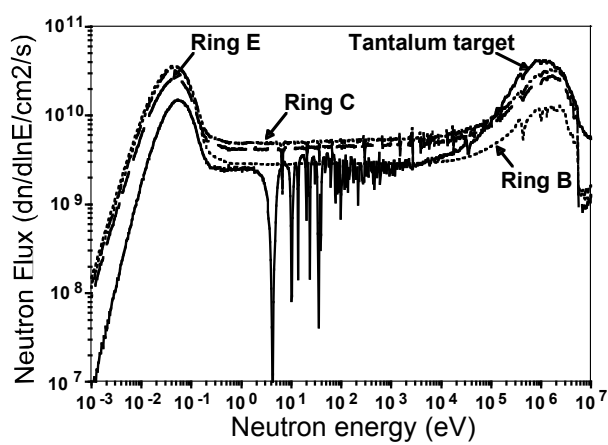

Figure 4: a) Example of the configuration of the TRIGA-ADS project b) Neutron flux spectra at selected locations of the TRIGA-ADS reference configuration [13].

\subsection{The principle of Piccolo-Micromegas}

The advantages of Micromegas over conventional neutron detector, described in the preceding paragraphs are used to extend the development for the measurement of neutron flux in a nuclear reactor core. Fissile elements such as ${ }^{235} \mathrm{U},{ }^{232} \mathrm{Th}$ are used simultaneously as neutron/charged particle converter in addition to ${ }^{10} \mathrm{~B}$ and recoil ions of the gas $\left(\mathrm{Ar}+\mathrm{iC}_{4} \mathrm{H}_{10}\right.$ quencher) filling the detector.

The Piccolo-Micromegas detector shown in Figure 5a is designed to cope with severe constraints encountered in a nuclear reactor environment (high radiation, high temperature, neutron activation, sealed detector). For this reason the detector structure, frames and sensitive elements have been carefully chosen and most of them are made out of stainless steel and ceramics. The detector consists of a drift electrode, a thin stainless steel cathode grid and four anode pads connected to fast amplifiers. The drift gap is $1 \mathrm{~mm}$ wide and the amplification gap $160 \mu \mathrm{m}$. In a second prototype, different amplification gaps were used in each compartment, in order to adapt the dynamic range of the collected charge. Because of the high reactor temperature and the high radiation yield, the electronics were placed outside and connected to the anode pads through special radiation hardened and low capacitance coaxial cables 10 meter long.

The drift electrode is composed of four neutron/charged particle converters: ${ }^{10} \mathrm{~B}$ for thermal and epithermal neutrons, ${ }^{235} \mathrm{U}$ for monitoring as the fission cross section of ${ }^{235} \mathrm{U}$ from thermal energies up to several $\mathrm{MeV}$ is well known, ${ }^{232} \mathrm{Th}$ with a fission threshold of about 1 $\mathrm{MeV}$ is dedicated to high energy neutrons. The elastic scattering of neutrons with light atoms 
from the gas filling the detector (hydrogen) will produce nuclear recoils than could be detected with a typical threshold of several $\mathrm{keV}$.

a)

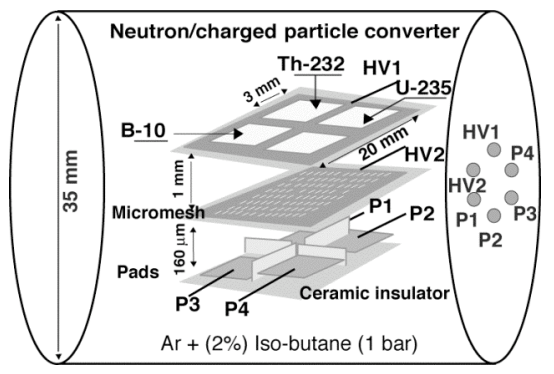

b)

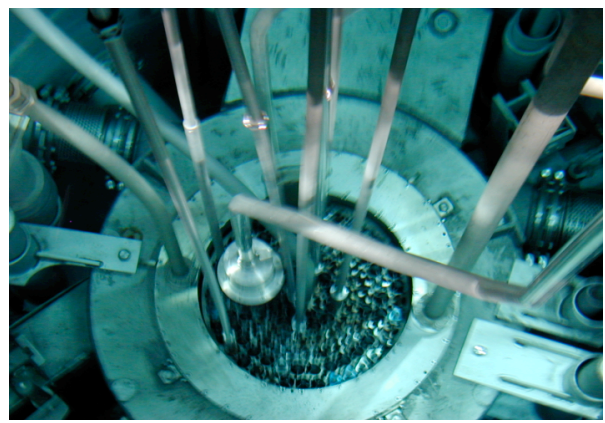

Figure 5: a) Schematic view of the principle of Piccolo-Micromegas detector (in horizontal position) for neutron flux measurement, b) View of the Piccolo-Micromegas assembly placed inside the TRIGA reactor

Using four converters with a unique detector will permit extracting practically on line a large range of the neutron flux spectrum in a specific position in the reactor. The large dynamic range of Piccolo-Micromegas will permit precise measurements and a detailed scanning of the flux into the whole reactor volume.

At very high counting rate $(>100 \mathrm{MHz})$ measurement will be performed on a current mode basis. At low counting rate, the fast response of the detector will allow counting one by one the incident particles using a low noise fast-preamplifier. It opens the way to measure the neutron flux at the peripheral part of the reactor and also in some cases when the full reactor power is not used.

\subsection{Tests and measurements with the first prototype with $14 \mathrm{MeV}$ neutron source}

A first test with sealed prototype of the detector has been performed with $14 \mathrm{MeV}$ neutrons given by the Cadarache CELINA facility.
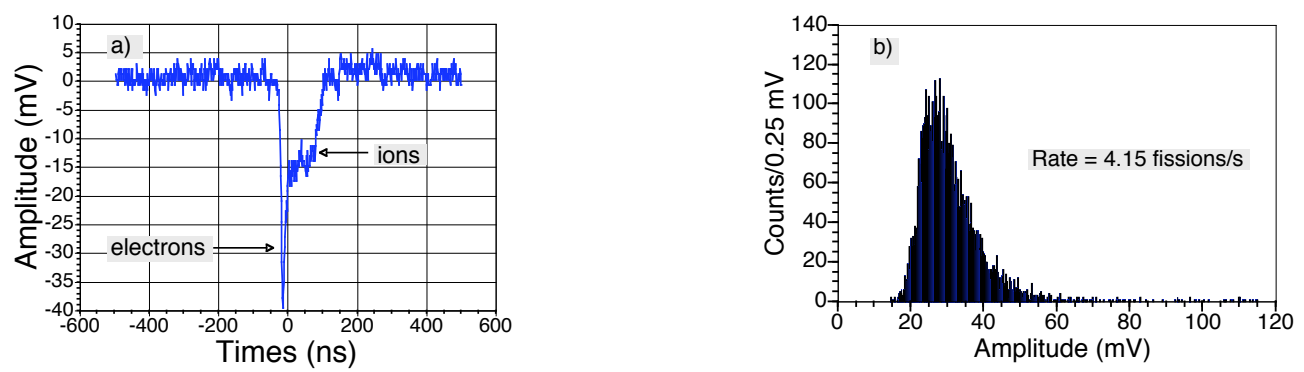

Figure 6: a) Example of the fission fragment pulse b) Amplitude spectrum of ${ }^{235} U$ fission fragments 
An example of the pulse obtained from the fission fragment emitted in the interaction of neutrons with ${ }^{235} \mathrm{U}$ is shown in figure 6a). This figure shows clearly the contribution of the detected electrons (fast part of the pulse) and ions (slow part) with a total width of $150 \mathrm{nsec}$.

An example of the spectrum of ${ }^{235} \mathrm{U}$ fission fragment as a function of the amplitude of the pulse is reported in figure 6b). A full simulation of the Piccolo-Micromegas detector placed inside the CELINA-Cadarache neutron source facility has been performed using FLUKA code [14]. The study has been centred on the particular case of ${ }^{235} \mathrm{U}$, because of the well-known fission cross section. For $50 \mu \mathrm{g}$ of ${ }^{235} \mathrm{U}$ the measured fission rate given in figure $6 \mathrm{~b}$ ) is in good agreement with the prediction (4 fissions/s).

\subsection{Tests and measurements with the first prototype in 1 MW TRIGA reactor}

A second test with sealed prototype placed inside an empty rod of the reactor has been performed in-core of the TRIGA reactor of ENEA at Casaccia (Italy). The detector was placed inside of a sealed long stainless tube having the same dimension of the reactor empty rod; usual $\mathrm{BNC}$ cables have been used and placed inside a $10 \mathrm{~m}$ watertight stainless tube (see Figure $5 \mathrm{~b}$ ). The measurement has been performed at two positions of the detector, at the periphery and in the centre of the reactor.

The characteristics of Piccolo-Micromegas (gain, field of the drift and ageing) per simultaneous measurement of the currents and the counting rates according to the power of the reactor were studied in detail. The preliminary results confirm that the detector such as it is conceived, is able to measure neutron flux from thermal to several $\mathrm{MeV}$ in the very aggressive environment of a reactor. It is possible with Micromegas to count event per event, the choice of the gain makes it possible to distinguish the neutrons of the gamma background. A linearity $(10 \%$ to $5 \%)$ of the neutron flux according to the power of the reactor has observed. Counting alpha, of radioactivity of ${ }^{235} \mathrm{U}$ before and after high power irradiation, seems to show that at the end of 10 days of operation in sealed condition, it was not observed of notable degradation of the detector.

\section{Conclusion}

The Micromegas technology already used for particle physics experiment has extended to develop a new neutron detector generation. The example reported in this paper show the ability of this type of neutron detector to operate in different environment conditions: in the air, in the vacuum, in the water.

As shown in this paper the new Piccolo-Micromegas can work in nuclear reactor, which is a very aggressive experimental condition.

Using four converters with a unique detector will permit to extract practically one line a large part of the neutron flux spectrum in dedicated position in the reactor. The ability of this type of detector to detect the high-energy neutron (via nucleus recoil detection) permit also to 
use it to monitor the impact of the proton beam on the spallation target for the ADS project. Such a system is very useful for safety.

Piccolo-Micromegas detector was studied for a particular case in the TRIGA-ADS project [13], but could be used in other applications for instance in actual or future generation nuclear reactors. Thanks to the innovative techniques used, the prototype detector actually designed and manufactured seems to meet the conditions for a correct operation in reactor.

\section{Acknowledgment}

The work related to Piccolo-Micromegas has been performed with the collaboration of:

S. Aune, A. Giganon, M. Riallot (CEA Saclay DSM/DAPNIA 91191 Gif-sur-Yvette, France), C. Blandin, S. Breaud, B. Geslot, C. Jammes (CEA/DEN/Cadarache, 13108 Saint-Paul Lez Durance-France), Y. Kadi, L. Sarchiapone (CERN CH 1211 Geneva-Switzerland), G. Ban, P. Laborie, J.F. Lecolley, J.C. Steckmeyer, J. Tillier (LPC - CNRS/IN2P3 - ENSICAEN - UCBN, 6 bd Maréchal Juin, 14050 Caen, France) , R. Rosa (ENEA-Casaccia, Via Anguillarese, 00060 Roma, Italy), G. Andriamonje (IXL - Université Bordeaux 1 F33405 Talence Cedex, France)

\section{References}

[1] I. Giomataris et al., "A high-granularity position sensitive gaseous detector for high particle-flux environments", Nucl. Instrum. Methods A 376 (1996) 29.

[2] I. Giomataris, Nucl. Instr. and Meth. A 419 (1998) 239.

[3] G. Charpak et al., Nucl. Instr. and Meth. A 478 (2002) 26.

[4] K.Zioutas et al., Phys. Rev. Letters, 94, 121301, (2005)

[5] S. Andriamonje et al., Nucl. Instr. Meth. A 481 (2002) 36

[6] S. Andriamonje et al., Advances in Neutron Scattering Instrumentation. Proceedings of SPIE, Volume 4785, Ian S. Anderson, Bruno Guýrard, Editors, November 2002, pp. 214-225

[7] J. Pancin, et al., Nucl. Instr. and Meth. A 524 (2004) 102

[8] P. Mandrillon, private communication

[9] J. Remillieux, private communication

[10] M. Houry et al., "DEMIN: a neutron spectrometer, Micromegas-type, for inertial confinement fusion experiments", Nucl. Instrum. Methods A 557 (2006) 648.

[11] S. Boyer et al., "A fast Neutron Detector for Nuclear Waste Characterization" Proceeding of GLOBAL 2005 Tsukuba, Japan (2005) Paper No. 303

[12] C.L. Britton et al., “A Detector for Neutron Imaging” IEEE (2004) 360

[13] The TRADE Working group, "TRiga Accelerator Driven Experiment (TRADE)" Feasibility Report. ENEA Report, March 2002.

[14] A. Fasso, et al., "Intermediate Energy Nuclear Data: Models and C", Proceedings of a Specialist's Meeting, Issy les Moulineaux (France) 1994

[15]C. Rubbia, et al., "Conceptual Design of a Fast Neutron Operated High Power Energy Amplifier", CERN/AT/95-44 (EET) Geneva, September 29, 1995. Y. Kadi, et al., "The EA-MC Monte Carlo Code Package", in Proc. of the 5th Int. Meeting on Simulating Accelerator Radiation Environment - SARE-5: Models and Codes for Spallation Neutron Sources, Paris, France, July 17-18, 2000.

[16] J. F. Briesmeister, Editor $A^{\prime \prime} \mathrm{MCNP}^{\mathrm{TM}}$, General Monte Carlo N-Particle Transport Code, Version 4C", Los Alamos National Laboratory report LA-13709-M, March 2000). 